$\S=-1$

\title{
Evaluation of Nature-Inspired Algorithms for Battery Parame-ter Estimation in Renewable Energy Generation Systems
}

\author{
Hussain Shareef ${ }^{1 *}$, Md. Mainul Islam², Samantha Sasi Stephen ${ }^{3}$ \\ ${ }^{1,3}$ Department of Electrical Engineering, United Arab Emirates University, 15551, 1 Al-Ain, UAE \\ ${ }^{2}$ School of Computing, Engineering and Mathematics, Western Sydney University, Locked Bag 1797, NSW 2751, Australia \\ *Corresponding author E-mail: shareef@uaeu.ac.ae
}

\begin{abstract}
Batteries in renewable energy systems suffer problems that affect their service life and quality of performance. Therefore, battery management systems (BMS) are employed in battery-integrated systems to maintain optimal operating conditions by various dynamic impedance based battery models. These models require an accurate model parameter for BMS to work effectively. In this paper, two recently developed metaheuristic optimization, namely bird mating optimizer (BMO) and lightning search algorithm (LSA) is used effectively to determine the required parameters of well-known Randle's battery model. Initially, electrochemical impedance spectroscopy (EIS) test for an EnerSys Cyclon lead-acid cell with a rated capacity of 2.5Ah is conducted using EZSTAT-pro Galvanostat/Potentiostat device from Nuvant systems Inc. Next, the Randle's battery model parameters are obtained by BMO and LSA and its performances are evaluated. The results show that BMO and LSA can accurately find the model parameters. LSA obtains slightly more accurate results than BMO and converges much faster. However, for the same number of iterations, BMO takes less computation time than LSA. The optimized model can be used in BMS for fault finding and condition monitoring.
\end{abstract}

Keywords: Bird mating optimizer (BMO); Electrochemical impedance spectroscopy (EIS); Lightning search algorithm (LSA); Randle's battery model.

\section{Introduction}

Batteries employed in renewable energy systems are multifunctional devices. The stochastic nature of power generation in renewable energy systems makes batteries imperative to ensure continuous power supply to the utility grid. Batteries basically function as a storage medium that absorbs excess power generated during the abundant availability of the renewable energy source. In times of shortages and unexpected system failures, batteries serve as a backup power supply. In grid interfaced renewable energy generation systems, batteries are critical to prevent grid instabilities. In stand-alone energy systems, battery energy storage (BES) also forms the 'backbone' module.

Batteries in some renewable energy systems suffer problems that affect their service life and quality of performance [1-6]. Battery management systems (BMS) are employed in battery integrated systems to maintain optimal operating conditions of the BES. The purpose of a typical BMS is to run periodical online and offline monitoring algorithms. These algorithms generally measure the values such as current, voltage, temperature. With these input, the model provides an estimate of various critical performance parameters of the battery. The BMS must also be equipped to implement the necessary correctives and control measures to prevent damage to the BES and enhance its service life.

Impedance-based monitoring is often employed for the analysis of BES modules due to its close relation to internal dynamic processes of a battery. Electrochemical impedance spectroscopy (EIS) is a widely prescribed tool for battery diagnosis as it provides very accurate insight into the chemical processes occurring in batteries
[7-9]. The technique involves the application of a small sinusoidal current or voltage signal to the battery and calculates its internal impedance based on the response signal. This technique is often implemented offline to capture a battery's charge transfer dynamics over a wide range of frequencies, typically ranging from $1 \mathrm{~Hz}$ to $100 \mathrm{kHz}$. EIS measurements coupled with equivalent circuit modelling (ECM) serves as a reliable technique for battery monitoring, state estimation and fault diagnosis [10-15].

\section{Impedance-Based Model}

Realizing a battery model that takes into account all the electrochemical dynamics inside a battery is a challenging task. Also, online BMS often offer small computational capacity, which requires limiting the number model parameters. But at the same time, the model must be able to effectively capture all the important underlying processes.

Three main electrochemical phenomena that govern the battery dynamics are the resistance offered to the charge carriers by the electrode, the transient behavior of the electrons that are transferred from the electrode to the electrolyte and the diffusive process of the electrons released to the electrolyte. A Thevenin-based model, known as the Randle's model is widely used as an equivalent circuit to model the aforementioned processes. A first-order Randle's model consists of a resistor $\mathrm{R}_{0}$ that represents the ohmic resistance of the electrodes, resistor $R_{1}$ that represents resistance encountered upon charge transfer from the electrode to the electrolyte and capacitor $\mathrm{C}_{1}$ that represents the double layer capacitance as shown in Figure 1. Many models incorporate multiple RC elements for im- 
proving the model accuracy. In this paper, a simple first-order model is used for analysis, with an inductor $\mathrm{L}$ added to represent the effect due to metallic interconnections and geometry of the cell. Since impedance measurements use an AC excitation signal, the addition of an inductor to the model becomes a requisite. To represent the diffusion process, a Warburg Impedance $Z_{\mathrm{WB}}$ represented by a series of infinite RC elements is used.

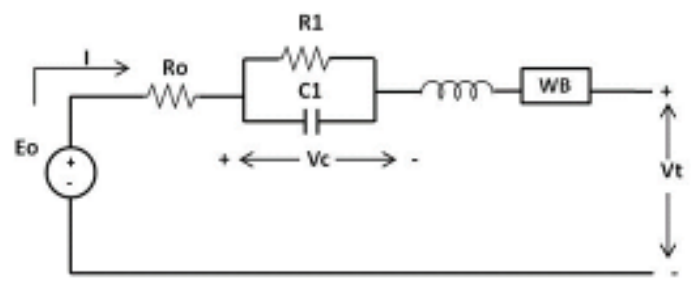

Fig.1: Typical equivalent circuit model for battery

A typical impedance spectrum for a battery cell known as the Nyquist plot is shown in Figure 2. Each part of the spectrum is attributed to a specific electrochemical phenomenon of the battery. The intercept made by the impedance spectrum on the real axis of the plot corresponds to $\mathrm{R}_{0}$. The semicircle that follows the intercept is a result of the transient reaction process due to $R_{1}$ and $C_{1}$. The straight line after the semicircle corresponds to the diffusion process which is prominent at lower frequencies. The negative tail that extends from the real axis intercept indicates the inductive behavior of battery which is prominent at very high frequencies. An input sinusoidal current signal $I(\omega)$, having an angular frequency $\omega$, when applied to the battery during the EIS test generates a response voltage signal $U$, from which the impedance of the cell under test can be obtained by

$Z_{B}(\omega)=\frac{U}{I(\omega)}<\Phi(\omega)$

where $\Phi(\omega)$ is the phase angle.

The impedance obtained can be expressed using a real part Z'and an imaginary part Z', as given by

$Z_{B}(\omega)=Z^{I}+j Z^{I I}$

From the Randle's circuit model, the impedance can be represented as

$Z_{B}(\omega)=R_{0}+j \omega L+\frac{1}{j \omega C_{1}+\frac{1}{R_{1}}}+Z_{W B}$

where $Z_{W B}=\frac{\sigma}{\sqrt{\omega}}(1-j) ; \quad \sigma=$ Warburg coefficient

A rearrangement of the equation (3) yields,

$Z_{B}(\omega)=\left[R_{0}+\frac{\frac{R_{1}}{\left(\omega C_{1}\right)^{2}}}{R_{1}^{2}+\left(\frac{1}{\omega C_{1}}\right)^{2}}+\frac{\sigma}{\sqrt{\omega}}\right]+j\left[\omega L-\frac{\frac{R_{1}^{2}}{\omega C_{1}}}{R_{1}^{2}+\left(\frac{1}{\omega C_{1}}\right)^{2}}-\frac{\sigma}{\sqrt{\omega}}\right]$

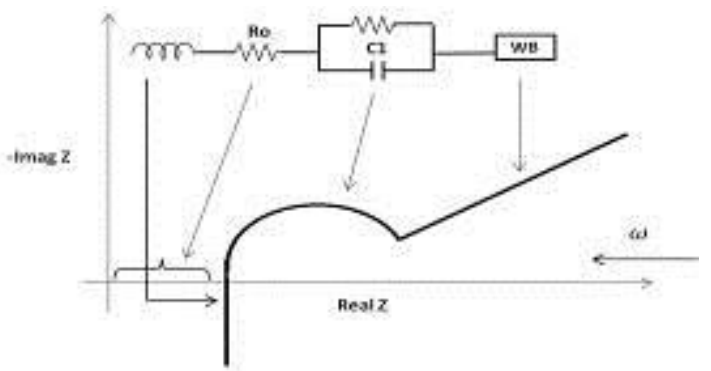

Fig. 2: Nyquist plot for battery cell

Parameter identification can be achieved through time domain or frequency domain parameter extraction methods [16]. The EIS test is the commonly used frequency-domain parameter extraction test. Compared to time-domain test, the amplitude of the excitation signal is so low that the battery can be considered equal during the whole process. By analyzing the impedance data obtained during EIS testing, a good estimation of the equivalent circuit model parameters can be found. The model details is achieved by determining the parameters that most accurately fit the measured impedance data to the Randle's model. Optimization algorithms are usually utilized to determine the best-fit. The focus of this paper is to evaluate the efficiency of two optimization algorithms in parameter estimation of Randle's model.

\section{Metaheuristic Algorithms}

Numerical optimization techniques have been widely used for finding global optimum solutions of real-world engineering problems. Some of the commonly used techniques include Maximum Likelihood Estimation (MLE) and Least Squares Method (LSM). However, in many real systems, there may exist more than one local optimum point which may not necessarily coincide with the global optimum. To tackle complex problems, researchers have investigated metaheuristic or evolutionary algorithms which combine rules with an element of randomness in order to mimic the natural phenomena observed in nature. Some of the phenomena that researchers have modelled include biological evolutionary processes [17-19] and the genetic algorithm [20-21], animal behavior [22], the physical annealing process [23] and the musical process of searching for a perfect state of harmony [24-28].

Every evolutionary algorithm goes through an exploration and exploitation process. Exploration refers to the generation of new solutions in untested regions of search space. Exploitation refers to the concentration of the search at the vicinity of the current good solution. The algorithm must be able to establish a reasonably good balance between these two processes in order to successfully converge to a good solution. Most metaheuristic algorithms are population-based. This means that the algorithm generates a pool of feasible candidate solutions, each solution corresponding to a unique point in the search space of the problem. The algorithm progresses by iteratively altering the current solutions to generate new ones. The candidates that provide a good solution to the problem, are retained for the next alteration process while the others are discarded. This process continues until the best solution is determined.

\subsection{Bird Mating Optimizer}

Bird mating optimizer (BMO) is a recently developed evolutionary algorithm that simulates commonly observed bird breeding strategies in nature [29]. In this algorithm, a society of birds forms the population that consists of male and female birds, with female birds being genetically superior. The breeding process is performed by the male bird combining his genes with the genes of a selected female bird to produce a new genetic brood. The selection of a female breeding mate is modeled using probabilistic techniques. The quality of each new bird that is produced via the breeding process is evaluated by means of the fitness function.

The algorithm models 5 types of breeding strategies namely monogamy, polygamy, polyandry, promiscuity and parthenogeny. The birds are divided into 5 species based on the type of breeding strategy they use. Each species has its own recombination method to produce genetically superior next-generation brood. This algorithm also incorporates a mutation operator to simulate the mutation process occurring during evolution, so as to improve the exploration process. Mutation is implemented in the algorithm by the male birds making a change in one of the brood's genes with a probability of 1 mutation control function (mcf).

For certain applications, the algorithm exhibited superior results compared to other similar metaheuristic algorithms [30, 31]. BMO has managed to establish a reasonable balance between the exploration and exploitation during the search space, which led to avoid 
unanticipated premature convergence to reach the global solution successfully.

In this paper, a simplified version of the original BMO algorithm has been used for the estimation of the battery ECM parameters from its impedance measurements. The first step of the algorithm is the initialization of a society with random birds having genes encoded as 5 ECM parameters of interest. Next, the birds of the society are ranked according to the fitness value. Then, these birds are classified into three different types according to predefined rank ranges, and the breeding process is performed accordingly. Finally, the quality of the brood of the previous generation is compared with the new generation using the specified fitness function, which is the root mean square error between the measured and estimated impedance value. The brood with higher fitness (i.e. lower root mean square error (RMSE)) is added to the society and the adjacent bird from the previous generation is discarded. The algorithm iterates until the stopping criterion is satisfied. Figure 3 shows the flowchart of BMO algorithm.

\subsection{Lightning Search Algorithm}

Lightning search algorithm (LSA) is a relatively new natureinspired algorithm, which models the phenomenon of lightning to solve complex optimization problems [32]. The emergence of a path of lightning strike termed as step leader and its propagation (step leader propagation) form the process flow of this algorithm. Conducting cloud-earth lightning channels develop as a result of the stochastic propagation of a step leader toward the earth. The major factor responsible for the dynamics of this process is the electric field strength near the leader tip. These step leaders progress to the earth by branching outwards in different directions in a series of steps. The leader that reaches the earth first provides the conducting channel between the earth and the cloud. Each of these leader tips possesses a certain energy, and they propagate by expending this energy to ionize the air in the vicinity of the tip. This algorithm is based on a formulated concept of fast particles known as projectiles which are assumed to be the initiators of a lightning strike. LSA is also a population-based algorithm with the first population of step leaders created by the ejection of $\mathrm{N}$ projectiles from a thunder cloud. Three types of projectiles are defined to model the step leader propagation mechanism.

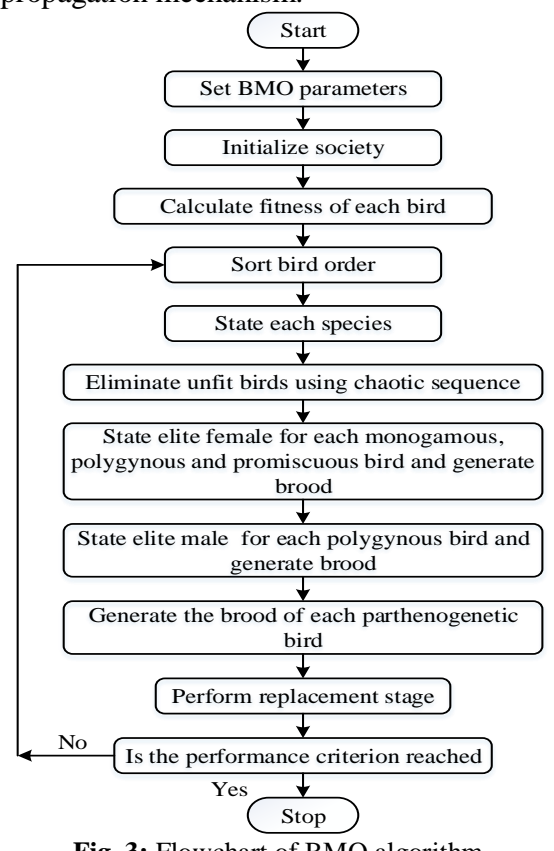

Fig. 3: Flowchart of BMO algorithm

The projectile that describes the transition of a projectile to a step leader is termed the transition projectile. Each of the projectiles that are ejected from the thunder cell collides with nearby particles to form a channel and hence a step leader. The emerging projectiles are modeled by random numbers drawn from a uniform distribu- tion in the open interval representing the solution space. These projectiles represent random candidate solutions for the optimization problem.

Once the $\mathrm{N}$ step leader tips have evolved, the leaders progress to the next step by ionizing the air in the vicinity of the old leader tip. The functional projectile in this phase is called the space projectile. The position of the space projectile in the new step is modeled as a random number generated from the exponential distribution with shaping parameter which controls the position or the direction of the space projectile. The new position does not guarantee step leader propagation or channel formation unless the projectile energy is greater than the step leader energy. If this criterion is met, then current step leader progresses to a new position in the next step and the leader that has extended the farthest will be the lead projectile.

The step leader that has travelled to reach nearest to the ground and the projectile associated with it will not have enough potential to ionize large sections in front of the leader tip. Therefore, the lead projectile can be modeled as a random number drawn from the standard normal distribution with a shape parameter and a scale parameter.

These properties that govern the collision kinematics of projectiles are used to design the exploitation and exploration phases of the algorithm. During the first stage of step leader propagation, their propagation is modelled using random distributions without any control in its direction or positions. This is to indicate the property of the newly created projectiles, have energies that can make them capable of ionizing a large space around them. This means that the algorithm can explore a large search space. But as they progress forward through the propagation steps, they gradually lose their energy due to repeated collisions, due to which they have only enough energy to ionize a small space. This means that the search space of the algorithm had now narrowed down to a small zone and the algorithm has switched to the exploitation phase. This phase is modelled by distributions that employ shaping and scaling parameters. A process known as forking is also programmed in the algorithm which represents the chance emergence of twin branches from a step leader. This algorithm had been successfully tested for optimizing controllers for photovoltaic applications [33].

For the battery parameter estimation problem, the boundary conditions for each parameter are given as inputs to the algorithm, and the first step leader population is initialized with projectiles fired at random directions encoded as the parameters of interest. Each of the step leaders, based on their fitness, form channels and pass through the various steps of propagation. The step leaders that provide the best solutions qualify to proceed to the next step, while the others are discarded. This iterative process continues until the algorithm finds the best solution. Figure 4 shows the flowchart of LSA technique.

\section{Optimization Fitness Function}

The target in parameter optimization is to minimize the fitness function for a number of iterations with respect to the parameters defined ranges. For obtaining the fitness function, the different between measured impedance and modeled impedance is obtained using equation (6) as:

$$
\begin{aligned}
& \mathrm{F}\left(Z_{B}, \omega\right)=Z_{B}(\text { measured })-Z_{B} \text { (model) } \\
& F\left(Z_{B}, \omega\right)=Z_{B}(\text { measured })-\left[\left[R_{0}+\frac{\frac{R_{1}}{\left(\omega C_{1}\right)^{2}}}{R_{1}^{2}+\left(\frac{1}{\omega C_{1}}\right)^{2}}+\frac{\sigma}{\sqrt{\omega}}\right]+j\left[\omega L-\frac{\frac{R_{1}^{2}}{\omega C_{1}}}{R_{1}^{2}+\left(\frac{1}{\omega C_{1}}\right)^{2}}-\frac{\sigma}{\sqrt{\omega}}\right]\right]
\end{aligned}
$$

where $Z_{B}$ (measured) represents the measured values of impedance from the EIS test, and $Z_{B}$ (model) is the fit impedance values generated using the ECM parameters estimated by the algorithms as shown in equation (6)

Then the RMSE is: 


$$
\sqrt{\frac{1}{N} \sum_{i=1}^{N}\left(\mathrm{~F}\left(Z_{B}, \omega\right)\right)^{2}}
$$

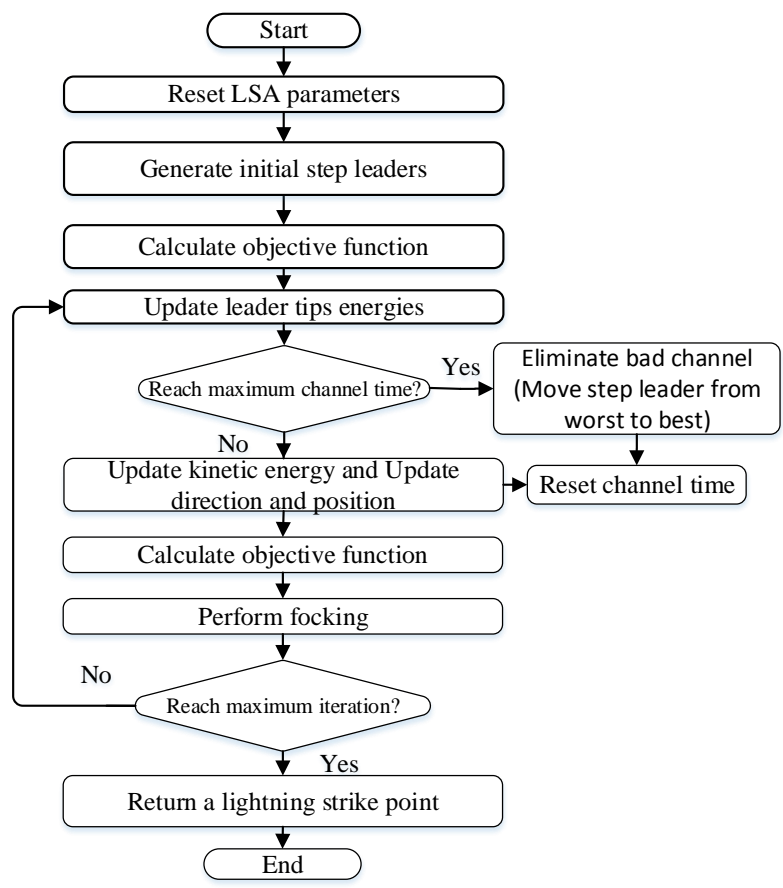

Fig. 4: Flowchart of LSA algorithm

\section{Experimental Setup}

The battery cell used for the test is an EnerSys Cyclon lead-acid cell with a rated capacity of $2.5 \mathrm{Ah}$. The EIS test was performed on the battery at nine different open-circuit voltage $\left(\mathrm{V}_{\mathrm{oc}}\right)$ levels. The battery was first fully charged, and then discharged to the desired $\mathrm{V}_{\mathrm{OC}}$ level using rated current. At the end of discharge, the cell is allowed a relaxation period to ensure the cell has reached equilibrium prior to conducting the test. Next, the EIS test was conducted by applying short-duration sinusoidal current signals of $10 \mathrm{~mA}$ for a frequency sweep in the range $0.01-8000 \mathrm{~Hz}$ to the test cell at room temperature. The EIS test was performed using EZSTATpro Galvanostat/Potentiostat device from Nuvant systems Inc (Figure 5). After the EIS tests, the cell is again discharged up to the next $V_{O C}$ level, and the same procedures are repeated for the nine $\mathrm{V}_{\mathrm{OC}}$ levels. The BMO and LSA algorithms have been coded in MATLAB environment.

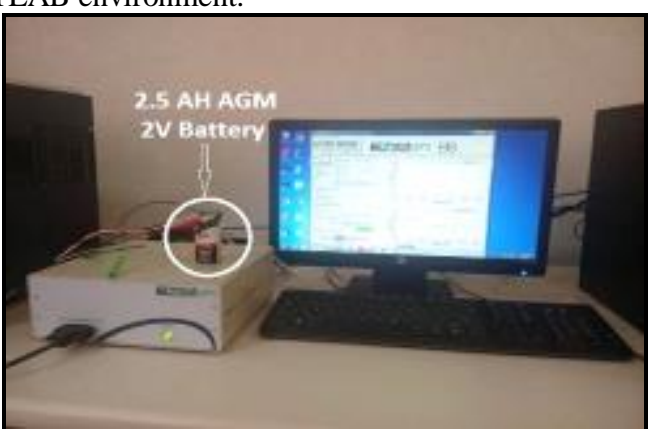

Fig. 5: Experimental test bench showing the testing device and lead-acid battery

\section{Results and Discussion}

The Bode plots and Nyquist plots were generated for nine $\mathrm{V}_{\mathrm{oc}}$ test levels of the cell. Figure 6 shows the Bode plot (a) which is the magnitude of impedance as a function of frequency and the Nyquist plot (b) plots the real part of impedance vs the imaginary part for voltage level $\mathrm{V}_{\mathrm{oc}}=2.02 \mathrm{~V}$. Each of the measured Nyquist curves were fit using a first order Randle's model consisting of a resistor $R_{0}, R_{1}, C_{1}, L$ and $\sigma$ which are the model parameters that are to be estimated. The LSA and BMO optimization algorithms were used to estimate the 5 parameters of interest as shown in Tables 1 and 2. The population size for both the algorithms was set to 50 and the initial values and boundary conditions were specified. The objective function used for optimization is the RMSE between the measured impedance data and the estimated impedance values using the model.

The performance of the algorithms can be studied with the help of the convergence characteristics. The convergence characteristics of the algorithm are represented using the fitness function value against the iteration number. In this case, the plot shows the RMSE value achieved at the end of each iteration of the algorithm. Figure 7 shows the convergence curve of the data in Figure 6. It can be seen that the both BMO and LSA algorithms were able to converge to the same accuracy. But the convergence characteristics indicated that the LSA algorithm exhibited a faster convergence within few iterations. For example, at the $200^{\text {th }}$ iteration, the LSA has reached a fitness value which has an error of $4.5 \%$ compared to final value. For the same number of iterations, BMO has reached a fitness value that is still 6 times the final value. The convergence pattern is consistent for all the Voc levels. The speeds of convergence of both the algorithms are generally good, but on the close comparison, it is seen that BMO takes lesser time to converge to the final value than LSA. For instance, for the same number of iterations for the data at $\mathrm{V}_{\mathrm{oc}}=2.089 \mathrm{~V}, \mathrm{BMO}$ converges within 14.874 seconds while LSA takes 34.564 seconds.

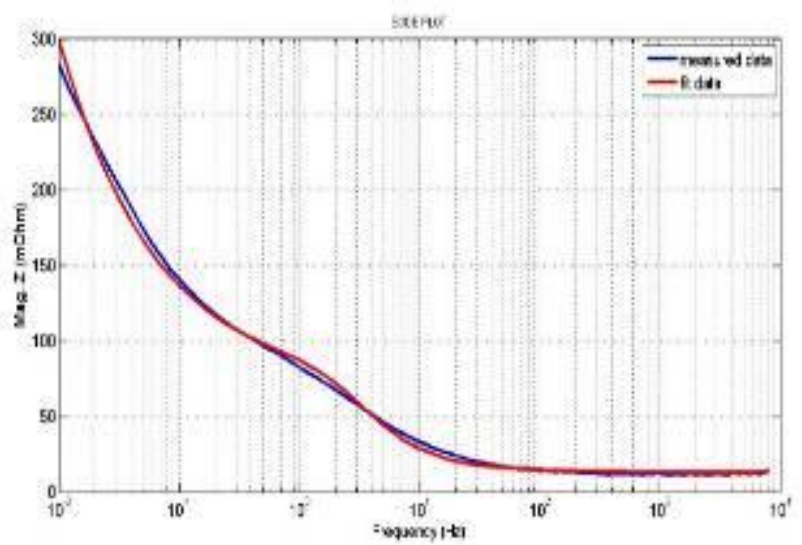

(a)

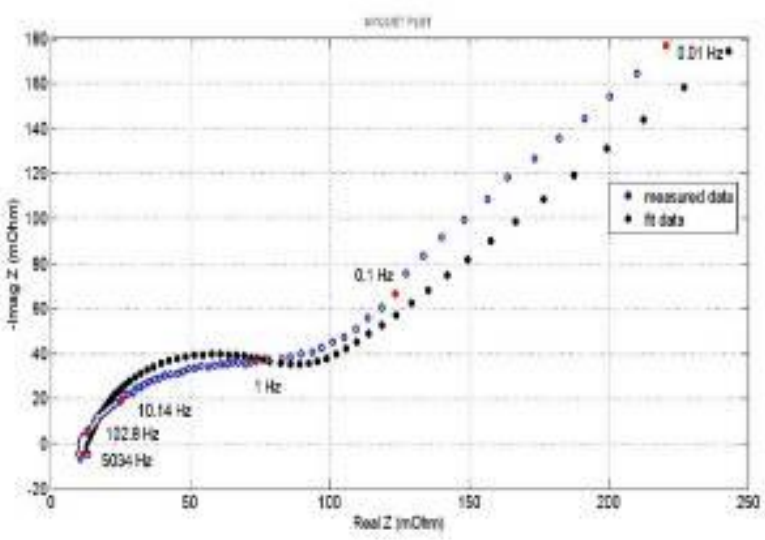

(b)

Fig. 6: (a) Bode plot, (b) Nyquist plot of the test cell measured at $\mathrm{V}_{\mathrm{oc}}=2.02 \mathrm{~V}$ 


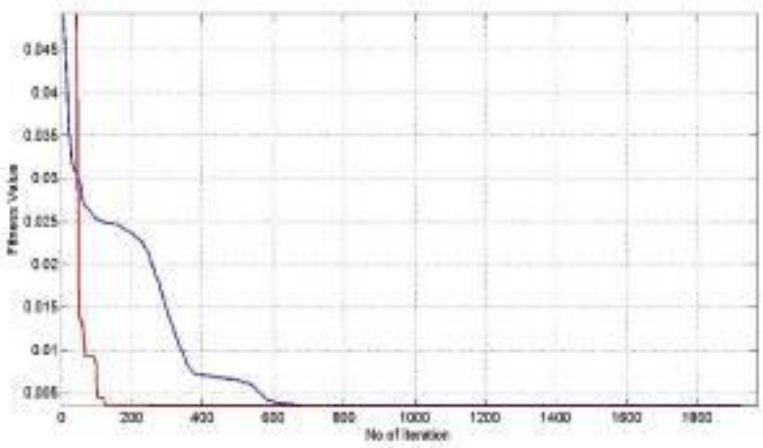

Fig. 7: Convergence characteristic of the test cell at Voc $=2.02 \mathrm{~V}$ (red: LSA, blue: $\mathrm{BMO})$

\section{Conclusion}

This paper evaluated the performance of two relatively new metaheuristic algorithms, BMO and LSA for optimal parameter estimation for the ECM of a battery cell. BMO models mating strategies adopted by bird species and LSA models the mechanism of lightning strike and propagation. The application of both algorithms for determining optimal estimates for the ECM of a battery cell is evaluated. The results show that both BMO and LSA are competent and exhibit a similar accuracy of parameter estimation for the first order Randle's model. However, LSA showed an advantage over BMO by possessing superior convergence characteristics. Also, there was a notable difference between the execution times of the algorithms, which must be investigated further.

Table 1: $2.5 \mathrm{AH} 2 \mathrm{~V}$ lead-acid cell equivalent circuit parameters fit using BMO algorithm

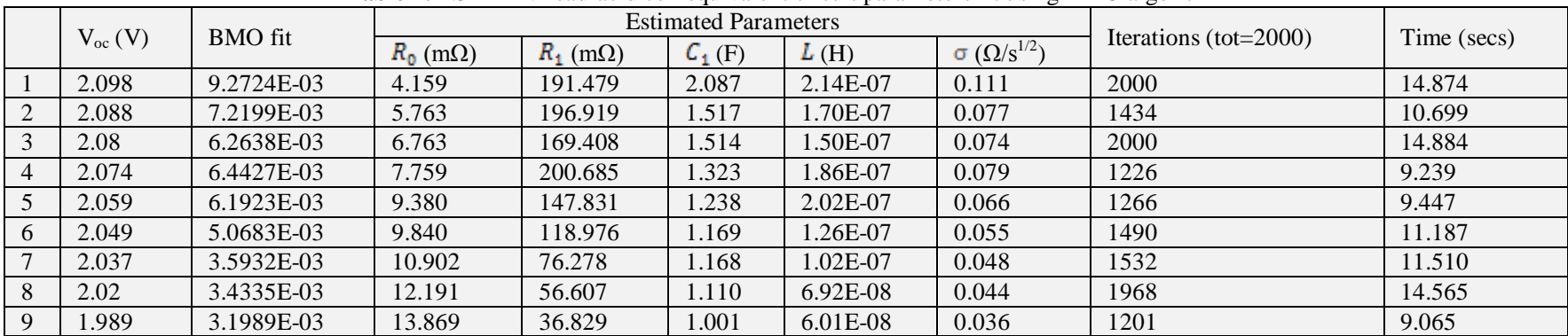

Table 2: 2.5 AH 2V lead-acid cell equivalent circuit parameters fit using LSA algorithm

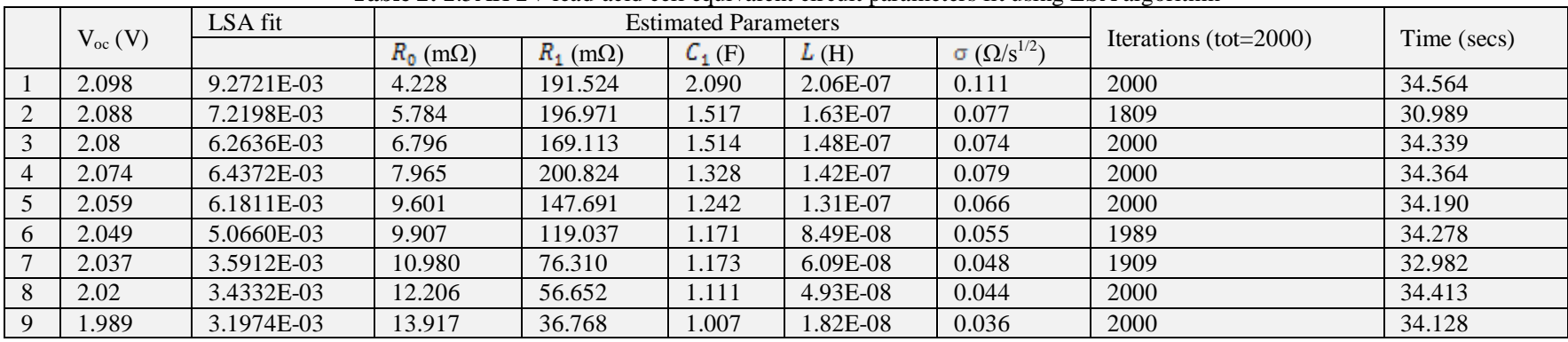

\section{References}

[1] Jossen A, Garche J \& Sauer DU (2004), Operation conditions of batteries in PV applications. Solar Energy 76, 759-769.

[2] Sauer DU, Bächler M, Bopp G, Höhe W, Mittermeier J, Sprau P Willer B \& Wollny M (1997), Analysis of the performance parameters of lead/acid batteries in photovoltaic systems. Journal of Power Sources 64, 1, 197-201.

[3] Ruetschi P (2004), Aging mechanisms and service life of leadacid batteries. Journal of Power Sources 127, 1-2, 33-44.

[4] Lam LT, Haigh NP, Phyland CG \& Urban AJ (2004), Failure mode of valve-regulated lead-acid batteries underhigh-rate partial-state-of-charge operation. Journal of Power Sources 133, $1,126-134$.

[5] Catherino HA, Feres FF \& Trinidad F (2004), Sulfation in leadacid batteries. Journal of Power Sources 129, 1, 113-120.

[6] Apăteanu L, Hollenkamp AF \& Koop MJ (1993), Electrolyte stratification in lead/acid batteries: Effect of grid antimony and relationship to capacity loss. Journal of Power Sources 46, 2-3, 239-250.

[7] Andre D, Meiler M, Steiner K, Walz H, Soczka-Guth T \& Sauer DU (2011), Characterization of high-power lithium-ion batteries by electrochemical impedance spectroscopy. II: Modelling. Journal of Power Sources 196, 12, 5349-5356.

[8] Ranieri M, Alberto D, Piret H \& Cattin V (2017), Electronic module for the thermal monitoring of a $\mathrm{Li}$-ion battery cell through the electrochemical impedance estimation. Microelectronics Reliability 79, 410-415.
[9] Tröltzsch U, Kanoun O \& Tränkler HR (2006), Characterizing aging effects of lithium ion batteries by impedance spectroscopy. Electrochimica Acta 51, 8-9, 1664-1672.

[10] Waag W, Käbitz S \& Sauer DU (2013), Experimental investigation of the lithium-ion battery impedance characteristic at various conditions and aging states and its influence on the application. Applied Energy 102, 885-897.

[11] Richardson RR, Ireland PT \& Howey DA (2014), Battery internal temperature estimation by combined impedance and surface temperature measurement. Journal of Power Sources 265, 254261.

[12] Gadsden SA \& Habibi SR (2011), Model-Based Fault Detection of a Battery System in a Hybrid Electric Vehicle. 2011 IEEE Vehicle Power and Propulsion Conference. Chicago, pp. 1-6.

[13] Brik K \& Ammar FB (2008), The Fault tree analysis of lead acid battery's degradation. Journal of Electrical Sytems 4, 2, 2-12.

[14] Westerhoff U, Kroker T, Kurbach K \& Kurrat M (2016), Electrochemical impedance spectroscopy based estimation of the state of charge of lithium-ion batteries. Journal of Energy Storage 8, 244-256.

[15] Křivík P (2018), Methods of SoC determination of lead acid battery. Journal of Energy Storage 15, 191-195.

[16] Böttiger M, Paulitschke M \& Bocklisch T (2017), Systematic experimental pulse test investigation for parameter identification of an equivalent based lithium-ion battery model. Energy Procedia 135, 337-346.

[17] Fogel LJ, Owens AJ \& Walsh MJ (1966), Artificial Intelligence Through Simulated Evolution. John Wiley, UK.

[18] Jong KD (1975), Analysis of the behavior of a class of genetic adaptive systems. Ph.D. Thesis. University of Michigan. Ann Arbor, MI. 
[19] Koza JR (1990), Genetic programming: a paradigm for genetically breeding populations of computer programs to solve problems. Stanford University, CA.

[20] Holland JH (1975), Adaptation in Natural and Artificial Systems. University of Michigan Press. Ann Arbor, MI.

[21] Goldberg DE (1989), Genetic Algorithms in Search, Optimization and Machine Learning. Addison Wesley, Boston.

[22] Glover F (1977), Heuristic for integer programming using surrogate constraints. Decision Sciences 8, 1, 156-166.

[23] Kirkpatrick S, Gelatt C \& Vecchi M (1983), Optimization by simulated annealing. Science 220, 671-680.

[24] Geem ZW, Kim JH \& Loganathan GV (2001), A new heuristic optimization algorithm: harmony search. Simulation 76, 2, 6068.

[25] Metropolis N, Rosenbluth AW, Rosenbluth MN, Teller AH \& Teller E (1953), Equations of state calculations by fast computing machines. A Journal of Chemical Physics 21, 1087-1092.

[26] Pincus M (1970), A Monte Carlo method for the approximate solution of certain types of constrained optimization problems. Operations Research 18, 1225-1228.

[27] Schwefel HP (1994), On the evolution of evolutionary computation. In: Computational Intelligence: Imitating Life, IEEE Press. New York, pp. 116-124.

[28] Kennedy J \& Eberhat RC (1995), Particle swarm optimization. In: Proceedings of IEEE International Conference on Neural Networks. IEEE Service Center, Piscataway, pp. 1942-1948.

[29] Askarzadeh A (2014), Bird mating optimizer: An optimization algorithm inspired by bird mating strategies. Communications in Nonlinear Science and Numerical Simulation 9, 4, 1213-1228.

[30] Omer ZM, Fardoun AA \& Hussein AA (2016), Large scale photovoltaic array fault diagnosis for optimized solar cell parameters extracted by heuristic evolutionary algorithm. IEEE PES General Meeting, USA.

[31] Zhu JJ, Huang M \& Lu ZR (2017), Bird mating optimizer for structural damage detection using a hybrid objective function. Swarm and Evolutionary Computation 35, 41-52.

[32] Shareef H, Ibrahim AA \& Mutlag AH (2015), Lightning search algorithm. Applied Soft Computing 36, 315-333.

[33] Shareef H, Mutlag AH \& Mohamed A (2015), A novel approach for fuzzy logic PV inverter controller optimization using lightning search algorithm. Neurocomputing 168, 435-453. 